\title{
Faixas de suficiência para teores foliares de nutrientes em algodão e em soja, definidas em função de índices DRIS ${ }^{1}$
}

\author{
Carlos Hissao Kurihara², Víctor Hugo Alvarez Venegas ${ }^{3}$, Júlio César Lima Neves ${ }^{4}$, \\ Roberto Ferreira de Novais ${ }^{5}$, Luiz Alberto Staut ${ }^{6}$
}

\section{RESUMO}

A diagnose dos estados nutricionais do algodoeiro e da soja, pelo método de níveis críticos, tem-se embasado em faixas de suficiência estabelecidas há muitos anos, com pequenas adequações no período. Contudo, considerando-se que os teores foliares podem variar, dentre outros fatores, em função do tipo de amostra coletada e do potencial produtivo da cultura, torna-se importante a definição de valores de referência regionais. Este trabalho teve como objetivo estabelecer os teores adequados de nutrientes para algodoeiro e soja, por meio do ajuste de modelos de regressão para o teor foliar em função do índice de equilíbrio nutricional definido pelo Sistema Integrado de Diagnose e Recomendação (DRIS). Utilizou-se um banco de dados, constituído das produtividades e dos teores de nutrientes em amostras foliares de ambas as espécies, coletadas em talhões de lavouras comerciais e em parcelas experimentais, em Mato Grosso do Sul e Mato Grosso. Foram obtidas faixas de suficiência para as folhas índice de algodoeiro (quinta folha totalmente formada, a partir do ápice) e de soja (terceiro trifólio sem e com pecíolo), com amplitude menor do que aquela estabelecida na literatura para estas culturas. Especificamente, para a soja, confirmou-se a existência de diferenças nos valores de referência em função do tipo de folha índice amostrado. Amostras de folha índice sem pecíolo produzem teores significativamente maiores de N, P, B, Fe, Mn e Zn e menores de K, em relação aos das amostras com pecíolo. A desconsideração do modo de amostragem pode induzir a falsos diagnósticos de deficiências ou excessos nutricionais.

Palavras-chave: análise foliar, sistema integrado de diagnose e recomendação, folha índice, Glycine max, Gossypium hirsutum.

\section{ABSTRACT \\ Sufficiency range for nutrient concentration in cotton and soybean leaves, defined through DRIS indexes}

Nutritional status diagnosis of cotton and soybean crops through critical level methods is based on sufficiency ranges established many years ago, with little advances since then. However, as nutrient contents in leaves are influenced by sample type and crop yield potential among other factors, it is important to establish regional reference values. The aim of this study was to establish optimum nutrient concentrations in cotton and soybean leaves by adjusting a regression model to nutrient concentration based on DRIS indexes. Database consisted of yield and nutrient content in leaf samples collected in commercial cotton and soybean crops and soybean experimental plots in

\footnotetext{
Recebido para publicação em 06/02/2013 e aprovado em 10/04/2013.

Parte da Tese de Doutorado do primeiro autor, apresentada ao Departamento de Solos da Universidade Federal de Viçosa. Trabalho financiado pela Embrapa/CNPq/Fundect. 2 Engenheiro-Agrônomo, Doutor. Embrapa Agropecuária Oeste, Rodovia BR 163, Km 253, 6, Caixa Postal 661, 79804-970, Dourados, Mato Grosso do Sul, Brasil. carlos.kurihara@embrapa.br (autor para correspondência)

${ }^{3}$ Engenheiro-Agrônomo, Doutor. Departamento de Solos, Universidade Federal de Viçosa, Campus Viçosa, Avenida Peter Henry Rolfs, s/n, 36570-000, Viçosa, Minas Gerais, Brasil.vhav@ufv.br

${ }^{4}$ Engenheiro-Agrônomo, Doutor. Departamento de Solos, Universidade Federal de Viçosa, Campus Viçosa, Avenida Peter Henry Rolfs, s/n, 36570-000, Viçosa, Minas Gerais, Brasil. julio_n2003@yahoo.com.br

${ }^{5}$ Engenheiro-Agrônomo, Doutor. Departamento de Solos, Universidade Federal de Viçosa, Campus Viçosa, Avenida Peter Henry Rolfs, s/n, 36570-000, Viçosa, Minas Gerais, Brasil.rfnovais@ufv.br

${ }^{6}$ Engenheiro-Agrônomo, Mestre. Embrapa Agropecuária Oeste, Rodovia BR 163, Km 253, 6, Caixa Postal 661, 79804-970, Dourados, Mato Grosso do Sul, Brasil. luiz.staut@embrapa.br
}

Rev. Ceres, Viçosa, v. 60, n.3, p. 412-419, mai/jun, 2013 
Mato Grosso do Sul and Mato Grosso States. On each selected place, chemical composition of leaves and crop production (whole cottonseeds or soybean grains) were evaluated at full blooming stage and physiological maturity, respectively. Based on the proposed methodology, sufficiency ranges for indexes leaves of cotton (fifth fully formed leaf) and soybean (third trifoliate leaf, without or with petiole) were determined, whith variation smaller than that presented in other papers reviewed. Specifically for the soybean crop, in addition to confirming the differences in the reference values according to the type of index leaf sampled. Samples without petiole produce significantly higher levels of N, P, B, Fe, Mn and Zn and lower K level, compared with samples with petiole. If disregarded, the manner of sampling can induce false diagnosis of nutritional deficiency or excesss in the crop.

Key words: foliar analysis, diagnosis and recommendation integrated system, index leaf, Glycine max, Gossypium hirsutum.

\section{INTRODUÇÃO}

Normalmente, os valores de níveis críticos e faixas de suficiência de nutrientes em tecidos vegetais são estabelecidos a partir de resultados de experimentos de calibração, em que o nutriente em estudo é aplicado em doses crescentes e os demais fatores de produção são supridos em quantidades adequadas e constantes (variáveis controladas constantes).

Quanto maior a estratificação de ambientes em que os experimentos são repetidos, como textura ou fator capacidade tampão, potencial produtivo ou sistema de manejo do solo, maior será a confiabilidade em se definir os valores de referência para dada cultura. Porém, como em geral não é conduzida uma vasta rede de experimentos, os valores de referência tornam-se válidos apenas para uma limitada amplitude de condições em que os fatores de produção foram considerados nos trabalhos de calibração. Além disto, o grau de insegurança pode ser aumentado quando se considera que, em algumas situações, os valores são definidos com certo grau de subjetividade, tomando-se como base a experiência do pesquisador; ou então, como no caso das culturas de algodoeiro e soja, os valores de referência adotados (Staut \& Kurihara, 2001; Embrapa Soja et al., 2010) foram estabelecidos em condições de potencial genético bastante diferentes dos verificados atualmente. Especificamente para a cultura da soja, as faixas de suficiência indicadas em Embrapa Soja et al. (2010) consistem, com pequenas alterações, nos valores estabelecidos no final da década de 80 por Sfredo et al. (1986), a partir das médias dos teores definidas para seis regiões norte-americanas produtoras de soja, apresentadas por Peck (1979).

Portanto, é plausível esperar que essas faixas de suficiência necessitem de adequações, considerando-se a existência de diferenças marcantes na eficiência em absorver e utilizar os nutrientes, bem como no potencial produtivo atualmente apresentado pelos cultivares. Em adição, mostra-se preocupante também o fato de as faixas de suficiência apresentarem amplitude demasiadamente grande, em que o limite superior chega a ser de cinco a 12 vezes maior do que o limite inferior, como no caso dos teores de Ca, Mn e Fe, em soja (Embrapa Soja et al., 2010), e de $\mathrm{Mn}, \mathrm{Fe}$, e Cu , em algodoeiro (Staut \& Kurihara, 2001).

Deve-se considerar, também, que não há consenso na literatura quanto ao procedimento na amostragem de folhas de plantas de soja, existindo a recomendação de coleta do terceiro trifólio a partir do ápice (Malavolta et al., 1997 e Embrapa Soja, 2002), bem como deste, acompanhado de pecíolos (Bataglia et al., 1996; Ribeiro et al., 1999). No entanto, apesar de o valor de referência para a diagnose do estado nutricional da soja ser influenciado pelo tipo de folha índice amostrado, tendo-se em vista haver maiores teores de $\mathrm{N}, \mathrm{P}, \mathrm{Cu}, \mathrm{Fe}, \mathrm{Mn}$ e $\mathrm{Zn}$ e menores de $\mathrm{K}$ no limbo foliar, em relação aos do terceiro trifólio com pecíolo (Kurihara, 2004), ainda são comumente adotadas faixas de suficiências únicas para a cultura.

Recentemente, têm surgido alternativas para o estabelecimento de faixas de suficiência, aproveitando-se de banco de dados provenientes de amostragens realizadas em talhões de fazendas, onde são avaliados, dentre outras variáveis, os teores de nutrientes nas folhas e a produtividade da cultura. A partir desse banco de dados, são aplicadas abordagens distintas dos princípios de métodos de diagnose do estado nutricional, no intuito de estimarem-se valores de referência associados a um dado potencial produtivo e, ou, condição de equilíbrio nutricional. Neste contexto, a estimativa dos valores de referência é feita por meio do relacionamento do teor foliar com o respectivo índice de equilíbrio do nutriente (Oliveira \& Cassol, 1995; Reis Júnior \& Monnerat, 2003; Kurihara, 2004; Urano et al., 2007; Serra et al., 2010; Harger, 2008), tomando-se como base o fato de que as faixas de suficiência estão relacionadas com uma amplitude de índice DRIS (Sistema Integrado de Diagnose e Recomendação) de $\pm 102 / 3$ desvios padrão (fator de ajuste $C=10$ ), ou seja, a faixa de equilíbrio $-6,67 \leq \mathrm{I} \leq+6,67$.

Rev. Ceres, Viçosa, v. 60, n.3, p. 412-419, mai/jun, 2013 
Com base no exposto, este trabalho teve como objetivo o estabelecimento de faixas de suficiência de teores foliares de nutrientes em algodoeiro e em soja, por meio do ajuste de modelos de regressão entre teores foliares de nutrientes e os respectivos índices DRIS, a partir de um banco de dados formado por amostras coletadas em 447 talhões de lavouras de algodoeiro e 608 talhões de lavouras e parcelas experimentais de soja.

\section{MATERIAL E MÉTODOS}

Entre 1998/99 e 2004/05, foram coletadas amostras de folhas índice de algodoeiro (quinta folha totalmente formada, a partir do ápice), em 447 talhões de lavouras, em 12 municípios de Mato Grosso do Sul e Mato Grosso. Para a cultura da soja, o banco de dados foi formado por 608 amostras de folhas índice (terceiro trifólio com pecíolo), coletadas em lavouras comerciais e em parcelas experimentais, em 26 municípios desses dois Estados, nos anos agrícolas de 1997/1998 a 2005/2006. As coletas foram efetuadas no estádio de floração plena de cada cultura, correspondente a R2 (Fehr \& Caviness, 1977), e entre F4 e F6 (Marur \& Ruano, 2001), para soja e algodoeiro, respectivamente. As amostras de tecido foliar foram submetidas à limpeza sequencial com água, solução ácida $(\mathrm{HCl} \mathrm{0,1}$ mol L ${ }^{-1}$ ) e água destilada. Após secagem em estufa de circulação forçada de ar, a $65^{\circ} \mathrm{C}$, por $72 \mathrm{~h}$, procedeu-se à moagem em moinho tipo Wiley (peneira $0,85 \mathrm{~mm}$ ). A análise química das amostras de tecido foliar foi efetuada conforme Malavolta et al. (1997). O N foi extraído por digestão sulfúrica e determinado pelo método semi-microKjeldahl. O P, K, Ca, Mg, S, Cu, Fe, Mn e Zn foram extraídos por digestão nítrico-perclórica e determinados por espectrometria de absorção molecular (P), fotometria de emissão de chama $(\mathrm{K})$, espectrofotometria de absorção atômica (Ca, Mg, Cu, Fe, Mn e Zn) e turbidimetria do sulfato de bário $(\mathrm{S})$. O B foi extraído por incineração e determinado por espectrometria de absorção molecular, pelo método da azometina $\mathrm{H}$.

No estádio de maturação fisiológica das culturas, correspondente a R8 (Fehr \& Caviness, 1977), e entre C9 e C11 (Marur \& Ruano, 2001), para soja e algodoeiro, respectivamente, efetuou-se a avaliação da produtividade de grãos de soja, corrigindo-se a umidade para $13 \%$, e de algodão em caroço, a partir da amostragem de três linhas de cultivo, com dois metros de comprimento cada, em quatro locais escolhidos aleatoriamente, no talhão onde se procedeu a coleta de amostras foliares.

A partir da população de referência, com produtividade superior a $3.750 \mathrm{~kg} \mathrm{ha}^{-1}$ de algodão em caroço (43,6 \% das amostras coletadas) e $3.600 \mathrm{~kg} \mathrm{ha}^{-1}$ de grãos de soja (34,2\% das amostras), foram estabelecidos os quocientes entre o teor de um dado nutriente (A) e os teores dos demais nutrientes $(\mathrm{B}, \mathrm{C}, \ldots \mathrm{N})$, sendo estes transformados em variáveis normais reduzidas (z) e aproximados a valores inteiros, pela multiplicação com o fator de ajuste (C). Para cada relação, foram calculadas as normais (média e desvio padrão). Pelo cálculo da média aritmética das relações diretas (A/B) e inversas (B/A), transformadas em variáveis normais reduzidas, foi definido o índice DRIS $\left(\mathrm{I}_{\mathrm{A}}\right)$, de acordo com Alvarez \& Leite (1999):

$$
\begin{aligned}
& Z(A / B)=[(A / B)-(a / b)](C / s) \\
& I_{A}=\frac{Z(A / B)+Z(A / C)+\ldots+Z(A / N)-Z(B / A)-Z(C / A)-\ldots-Z(N / A)}{2(n-1)}
\end{aligned}
$$
em que,

$\mathrm{C}=10$ = fator de ajuste;

$\mathrm{A} / \mathrm{B}$ e $\mathrm{a} / \mathrm{b}=$ relação dual entre os teores de nutrientes ( $\mathrm{g}$ $\mathrm{kg}^{-1}$ e $\mathrm{mg} \mathrm{kg}^{-1}$, para macro e micronutrientes, respectivamente) na amostra e na população de referência, respectivamente;

$\mathrm{s}=$ desvio padrão da relação dual da população de referência;

$\mathrm{n}=$ número de nutrientes envolvidos na análise.

Para a determinação da faixa de suficiência de nutrientes, os teores foliares da população de referência e da população de baixa produtividade (total de 447 amostras de folhas índice de algodoeiro e 608 amostras de folhas índice de soja) foram relacionados com os respectivos índices DRIS, conforme Oliveira \& Cassol (1995), Reis Júnior \& Monnerat (2003), Kurihara (2004) e Harger (2008). Uma vez estabelecido o modelo de regressão com melhor ajuste entre estas variáveis, definiu-se como faixa normal os teores foliares estimados, considerando-se uma amplitude de índice DRIS de $-6,67 \leq 1 \leq 0,00$.

Em 96 amostras foliares de soja, coletadas em cinco municípios de Mato Grosso do Sul, nos anos agrícolas de 2001/2002 a 2004/2005, procedeu-se à análise química do limbo foliar e do pecíolo, separadamente, com o intuito de se avaliar a relação entre teores de nutrientes no trifólio, na ausência e na presença do pecíolo.

\section{RESULTADOS E DISCUSSÃO}

Os teores de nutrientes em folhas de algodoeiro e de soja apresentaram ajustes significativos de modelo linear, quadrático ou exponencial, quando relacionados com os Índices DRIS (Tabelas 1 e 2, respectivamente), sendo que, para ambas as culturas avaliadas, constatou-se maior dispersão dos dados para o $\mathrm{N}$, resultando em menores coeficientes de determinação. Esperava-se ajuste de modelo linear para todos os nutrientes, porém, as curvaturas observadas na dispersão de pontos são, provavelmente, decorrentes de desvios de simetria na distribuição das relações duais (Kurihara, 2004). 
O ajuste de um modelo de regressão para estas variáveis baseia-se no fato de que teores foliares associados à faixa de equilíbrio para os índices DRIS (- 6,67 e 6,67, o que inclui $50 \%$ da população ao redor da média) correspondem à faixa normal de Beaufils (1973). Para amostras foliares com teores inferiores ou superiores a esta faixa de suficiência, os índices DRIS indicariam possível comprometimento de produtividade, por deficiência ou excesso nutricional, respectivamente (Reis Júnior, 2002), razão pela qual são definidos como limites para as faixas de interpretação deficiente ou em excesso. No intuito de se evitar a indução de desequilíbrios nutricionais, optouse, neste trabalho, por restringir a faixa suficiente, para teores foliares associados à amplitude, de 10 2/3 desvios padrão (fator de ajuste $\mathrm{C}=10$ ), ou seja, $-6,67 \leq 1 \leq 0,0$, pelo que teores de nutrientes relacionados com índice DRIS inferiores a - 6,67, no intervalo entre 0,0 e 6,67 e maiores do que 6,67 , foram definidos como baixo, alto e em excesso, respectivamente.
Tanto para o algodoeiro (Tabela 1), como para a soja (Tabela 2), observa-se que a amplitude dos valores de índice DRIS estabelecidos para as amostras componentes do banco de dados, é bastante abrangente, indicando haver representatividade de talhões, onde a produtividade efetivamente foi limitada por deficiência ou por excesso nutricional. Assim, a partir das equações de regressões apresentadas nas Tabelas 1 e 2, foram estimados os teores foliares de nutrientes considerados deficiente, suficiente, alto e em excesso, para fins de diagnose do estado nutricional do algodoeiro e da soja (Tabelas 3 e 4, respectivamente).

Na Tabela 3, verifica-se que, para o algodoeiro, as amplitudes das faixas de teores de nutrientes estimadas como suficiente são consideravelmente inferiores àquelas estabelecidas em Staut \& Kurihara (2001). Esta diminuição foi mais pronunciada para $\mathrm{Mn}(92 \%), \mathrm{Fe}(90 \%)$, $\mathrm{Mg}(86 \%)$ e $\mathrm{Cu}(80 \%)$, sendo que a diferença entre os valores definidos em Staut \& Kurihara (2001) para o limite

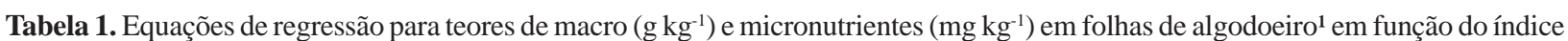
DRIS 2 , em amostras coletadas em Mato Grosso do Sul e Mato Grosso

\begin{tabular}{|c|c|c|}
\hline \multicolumn{2}{|c|}{ Equação } & \multirow{2}{*}{$\frac{\mathbf{R}^{2}}{0,351}$} \\
\hline$\hat{\mathrm{N}}=43,2+0,617 * * \mathrm{I}_{\mathrm{N}}$ & $-13,8 \leq \mathrm{I}_{\mathrm{N}} \leq 20,6$ & \\
\hline$\hat{\mathrm{P}}=2,79+0,0844 * * \mathrm{I}_{\mathrm{P}}+0,00081 * * \mathrm{I}_{\mathrm{P}}{ }^{2}$ & $-37,5 \leq \mathrm{I}_{\mathrm{P}} \leq 41,7$ & 0,674 \\
\hline$\widehat{\mathrm{K}}=18,24 \mathrm{e}^{0,0427 * *} \mathrm{~K}$ & $-32,3 \leq \mathrm{I}_{\mathrm{K}} \leq 17,3$ & 0,737 \\
\hline$\widehat{\mathrm{Ca}}=20,78 \mathrm{e}^{0,0308^{* *} \mathrm{Ca}}$ & $-35,6 \leq \mathrm{I}_{\mathrm{Ca}} \leq 23,2$ & 0,662 \\
\hline $\mathrm{Mg}=3,360 \mathrm{e}^{0,0313 * * \mathrm{I}} \mathrm{Mg}$ & $-38,9 \leq \mathrm{I}_{\mathrm{Mg}} \leq 39,1$ & 0,692 \\
\hline$\hat{\mathrm{S}}=5,369 \mathrm{e}^{0,0524 * * \mathrm{I}} \mathrm{S}$ & $-33,6 \leq \mathrm{I}_{\mathrm{S}} \leq 22,1$ & 0,861 \\
\hline$\hat{\mathrm{B}}=37,89 \mathrm{e}^{0,0474 * \mathrm{I}} \mathrm{B}$ & $-48,0 \leq \mathrm{I}_{\mathrm{B}} \leq 34,2$ & 0,841 \\
\hline$\hat{\mathrm{C}} \mathrm{u}=10,7+0,644 * * \mathrm{I}_{\mathrm{Cu}}+0,0103 * * \mathrm{I}_{\mathrm{Cu}}{ }^{2}$ & $-52,1 \leq \mathrm{I}_{\mathrm{Cu}} \leq 27,1$ & 0,912 \\
\hline$\hat{\mathrm{Fe}}=69,74 \mathrm{e}^{0,0485 * * \mathrm{~F}} \mathrm{Fe}$ & $-46,7 \leq \mathrm{I}_{\mathrm{Fe}} \leq 34,3$ & 0,897 \\
\hline$\hat{\mathrm{Mn}}=65,32 \mathrm{e}^{0,0602 * * \mathrm{I}} \mathrm{Mn}$ & $-29,4 \leq \mathrm{I}_{\mathrm{Mn}} \leq 26,8$ & 0,872 \\
\hline$\hat{Z}_{n}=26,56 \mathrm{e}^{0,0653^{* *} \mathrm{In}} \mathrm{Zn}$ & $-34,0 \leq \mathrm{I}_{\mathrm{Zn}} \leq 27,7$ & 0,918 \\
\hline
\end{tabular}

${ }^{1}$ Quinta folha totalmente formada, a partir do ápice, no ramo vegetativo da planta, coletada no estádio de floração plena. ${ }^{2}$ Modelos ajustados a partir de 447 amostras foliares coletadas em talhões de lavouras, em 12 municípios de Mato Grosso do Sul e Mato Grosso, no período entre 1998/99 e 2004/05.

Tabela 2. Equações de regressão para teores de macro $\left(\mathrm{g} \mathrm{kg}^{-1}\right)$ e micronutrientes $\left(\mathrm{mg} \mathrm{kg}^{-1}\right)$ no trifólio de soja com pecíolo em função do índice DRIS², em amostras coletadas em Mato Grosso do Sul e Mato Grosso

\begin{tabular}{llc}
\hline & Equação & $\mathbf{R}^{2}$ \\
\hline$\hat{N}=41,8+0,758 * * \mathrm{I}_{\mathrm{N}}$ & $-23,0 \leq \mathrm{I}_{\mathrm{N}} \leq 21,6$ & 0,567 \\
$\hat{\mathrm{P}}=2,77+0,078 * * \mathrm{I}_{\mathrm{P}}+0,002 * * \mathrm{I}_{\mathrm{P}}{ }^{2}$ & $-19,5 \leq \mathrm{I}_{\mathrm{P}} \leq 20,8$ & 0,698 \\
$\hat{\mathrm{K}}=21,2+0,631 * * \mathrm{I}_{\mathrm{K}}+0,006 * \mathrm{I}_{\mathrm{K}}{ }^{2}$ & $-23,8 \leq \mathrm{I}_{\mathrm{K}} \leq 20,3$ & 0,743 \\
$\hat{\mathrm{C}} \mathrm{a}=9,29+0,373 * * \mathrm{I}_{\mathrm{Ca}}$ & $-16,3 \leq \mathrm{I}_{\mathrm{Ca}} \leq 18,6$ & 0,729 \\
$\mathrm{M} \mathrm{g}=3,67+0,138 * * \mathrm{I}_{\mathrm{Mg}}+0,002 * * \mathrm{I}_{\mathrm{Mg}}{ }^{2}$ & $-17,6 \leq \mathrm{I}_{\mathrm{Mg}} \leq 21,1$ & 0,753 \\
$\hat{\mathrm{S}}=2,60+0,068 * * \mathrm{I}_{\mathrm{S}}$ & $-33,6 \leq \mathrm{I}_{\mathrm{S}} \leq 25,7$ & 0,686 \\
$\hat{\mathrm{B}}=41,1+1,27 * * \mathrm{I}_{\mathrm{B}}+0,008 * * \mathrm{I}_{\mathrm{B}}{ }^{2}$ & $-27,3 \mathrm{I}_{\mathrm{B}} \leq 22,3$ & 0,803 \\
$\hat{\mathrm{C}} \mathrm{u}=8,11+0,407 * * \mathrm{I}_{\mathrm{Cu}}+0,005 * * \mathrm{I}_{\mathrm{Cu}}{ }^{2}$ & $-26,0 \leq \mathrm{I}_{\mathrm{Cu}} \leq 37,9$ & 0,874 \\
$\hat{\mathrm{F}}=85,9+4,59 * * \mathrm{I}_{\mathrm{Fe}}+0,077 * * \mathrm{I}_{\mathrm{Fe}}{ }^{2}$ & $-22,0 \leq \mathrm{I}_{\mathrm{Fe}} \leq 37,5$ & 0,931 \\
$\hat{\mathrm{M}} \mathrm{n}=47,9+3,52 * * \mathrm{I}_{\mathrm{Mn}}+0,086 * * \mathrm{I}_{\mathrm{Mn}}{ }^{2}$ & $-29,1 \leq \mathrm{I}_{\mathrm{Mn}} \leq 23,2$ & 0,942 \\
$\hat{\mathrm{Z}} \mathrm{n}=42,4+2,08 * * \mathrm{I}_{\mathrm{Zn}}+0,048 * * \mathrm{I}_{\mathrm{Zn}}{ }^{2}$ & $-28,7 \leq \mathrm{I}_{\mathrm{Zn}} \leq 21,0$ & 0,843 \\
\hline
\end{tabular}

${ }^{1}$ Terceiro trifólio totalmente formado, a partir do ápice, no ramo vegetativo da planta, coletado no estádio de floração plena. ${ }^{2}$ Modelos ajustados a partir de 608 amostras foliares coletadas em lavouras comerciais e parcelas experimentais, em 26 municípios de Mato Grosso do Sul e Mato Grosso, nos anos agrícolas de 1997/1998 a 2005/2006. 
superior e inferior da faixa considerada suficiente, que chegava a 12 vezes para o $\mathrm{Mn}$ (entre 25 e $300 \mathrm{mg} \mathrm{kg}^{-1}$ ), foi reduzida para $1,5 \mathrm{vez}$ (entre $44 \mathrm{e} 65 \mathrm{mg} \mathrm{kg}^{-1}$ ) com o uso dos critérios propostos.

Da mesma forma, para a soja (Tabela 4), as amplitudes das faixas de teores estimados como suficientes em amostras de folhas com pecíolo, também são inferiores àquelas estabelecidas em Embrapa Soja et al. (2010) para limbo foliar. A redução da amplitude foi mais evidente para $\mathrm{Fe}$ (91\%), $\mathrm{Mg}(89 \%)$ e Ca (85\%), sendo que, no caso do Fe, o limite superior da faixa de teores suficientes, estabelecida em Embrapa Soja et al. (2010), supera o limite inferior em sete vezes (entre 50 e $350 \mathrm{mg} \mathrm{kg}^{-1}$ ) e a diferença para os valores estimados neste trabalho é de apenas $1,5 \mathrm{vez}$ (entre $59 \mathrm{e} 86 \mathrm{mg} \mathrm{kg}^{-1}$ ).

Resultados semelhantes foram obtidos por Harger (2008), que constatou, para amostras de folhas de soja coletadas em diferentes regiões produtoras do Paraná, redução na amplitude de valores da faixa suficiente, estimados conforme Kurihara (2004), em relação ao estabelecido em Embrapa Soja et al. (2008). Para as condições de lavouras de soja, cultivadas em solos de origem basáltica, este autor verificou estreitamento na faixa suficiente de todos os nutrientes, à exceção do $\mathrm{N}$ e principalmente para $\mathrm{B}, \mathrm{Mg}$,

Tabela 3. Teores de nutrientes estabelecidos ${ }^{1}$ para a cultura do algodoeiro e estimados ${ }^{2}$ pelo método Índice DRIS, para a interpretação dos resultados da análise foliar

\begin{tabular}{|c|c|c|c|c|c|}
\hline \multirow{2}{*}{ Nutriente } & \multirow{2}{*}{$\begin{array}{c}\text { Estabelecida }^{1} \\
\text { Suficiente }\end{array}$} & \multicolumn{4}{|c|}{ Estimada $^{2}$} \\
\hline & & Baixo & Suficiente & Alto & Excesso \\
\hline & \multicolumn{5}{|c|}{$\mathrm{g} \mathrm{kg}^{-1}$} \\
\hline $\mathrm{N}$ & 35 a 43 & $<39,1$ & 39,1 a 43,2 & 43,3 a 47,3 & $>47,3$ \\
\hline $\mathrm{P}$ & 2,5 a 4,0 & $<2,3$ & 2,3 a 2,8 & 2,9 a 3,4 & $>3,4$ \\
\hline K & 15 a 25 & $<13,7$ & 13,7 a 18,2 & 18,3 a 24,2 & $>24,2$ \\
\hline $\mathrm{Ca}$ & 20 a 35 & $<16,9$ & 16,9 a 20,8 & 20,9 a 25,5 & $>25,5$ \\
\hline $\mathrm{Mg}$ & 3 a 8 & $<2,7$ & 2,7 a 3,4 & 3,5 a 4,1 & $>4,1$ \\
\hline \multirow[t]{2}{*}{$\mathrm{S}$} & 4 a 8 & $<3,8$ & 3,8 a 5,4 & 5,5 a 7,6 & $>7,6$ \\
\hline & \multicolumn{5}{|c|}{$\mathrm{mg} \mathrm{kg}^{-1}$} \\
\hline B & 30 a 50 & $<28$ & 28 a 38 & 39 a 52 & $>52$ \\
\hline $\mathrm{Cu}$ & 5 a 25 & $<7$ & 7 a 11 & 12 a 15 & $>15$ \\
\hline $\mathrm{Fe}$ & 40 a 250 & $<50$ & 50 a 70 & 71 a 96 & $>96$ \\
\hline $\mathrm{Mn}$ & 25 a 300 & $<44$ & 44 a 65 & 66 a 98 & $>98$ \\
\hline $\mathrm{Zn}$ & 25 a 60 & $<17$ & 17 a 27 & 28 a 41 & $>41$ \\
\hline
\end{tabular}

${ }^{1}$ Staut \& Kurihara (2001); ${ }^{2}$ Faixa suficiente estimada a partir de ajuste de modelo de regressão para teores de nutrientes em folhas de algodoeiro em função do índice DRIS, calculado conforme Alvarez V. \& Leite (1999), considerando-se uma amplitude de - 6,67 I $\leq 0$.

Tabela 4. Teores de nutrientes estabelecidos ${ }^{1}$ para a cultura da soja (folha sem pecíolo) e estimados ${ }^{2}$ (folhas com pecíolo) pelo método Índice DRIS, para a interpretação dos resultados da análise foliar

\begin{tabular}{|c|c|c|c|c|c|}
\hline \multirow{2}{*}{ Nutriente } & \multirow{2}{*}{$\begin{array}{c}\text { Estabelecida }^{1} \\
\text { Suficiente }\end{array}$} & \multicolumn{4}{|c|}{ Estimada $^{2}$} \\
\hline & & Baixo & Suficiente & Alto & Excesso \\
\hline & \multicolumn{5}{|c|}{$\mathrm{g} \mathrm{kg}^{-1}$} \\
\hline $\mathrm{N}$ & 45,0 a 55,0 & $<36,8$ & 36,8 a 41,8 & 41,9 a 46,9 & $>46,9$ \\
\hline $\mathrm{P}$ & 2,5 a 5,0 & $<2,3$ & 2,3 a 2,8 & 2,9 a 3,4 & $>3,4$ \\
\hline K & 17,0 a 25,0 & $<17,3$ & 17,3 a 21,2 & 21,3 a 25,7 & $>25,7$ \\
\hline $\mathrm{Ca}$ & 3,5 a 20,0 & $<6,8$ & 6,8 a 9,3 & 9,4 a 11,8 & $>11,8$ \\
\hline $\mathrm{Mg}$ & 2,5 a 10,0 & $<2,9$ & 2,9 a 3,7 & 3,8 a 4,7 & $>4,7$ \\
\hline \multirow[t]{2}{*}{ S } & 2,0 a 4,0 & $<2,1$ & 2,1 a 2,6 & 2,7 a 3,0 & $>3,0$ \\
\hline & \multicolumn{5}{|c|}{$\mathrm{mg} \mathrm{kg}^{-1}$} \\
\hline $\bar{B}$ & 20 a 55 & $<33$ & $33 a 41$ & 42 a 50 & $>50$ \\
\hline $\mathrm{Cu}$ & 6 a 14 & $<6$ & 6 a 8 & 9 a 11 & $>11$ \\
\hline $\mathrm{Fe}$ & 50 a 350 & $<59$ & 59 a 86 & 87 a 120 & $>120$ \\
\hline $\mathrm{Mn}$ & 20 a 100 & $<28$ & 28 a 48 & 49 a 75 & $>75$ \\
\hline $\mathrm{Zn}$ & 20 a 50 & $<31$ & 31 a 42 & 43 a 58 & $>58$ \\
\hline
\end{tabular}

${ }^{1}$ Embrapa Soja et al. (2010); ${ }^{2}$ Faixa suficiente estimada para o terceiro trifólio totalmente formado, a partir do ápice, no ramo vegetativo da planta, coletado no estádio de floração plena, a partir de ajuste de modelo de regressão para teores de nutrientes em folhas de soja em função do índice DRIS, calculado conforme Alvarez V. \& Leite (1999), considerando-se uma amplitude de - 6,67 $\leq$ I $\leq 0$.

Rev. Ceres, Viçosa, v. 60, n.3, p. 412-419, mai/jun, 2013 
Ca e Fe. Salienta-se que os limites superiores da faixa suficiente, estimados por Kurihara (2004) e Harger (2008), para amostras de trifólios com pecíolo, na cultura da soja, apresentam em geral valores próximos aos limites superiores da classe de teor considerada alta (Tabela 4). Isto já era esperado, visto que aqueles autores consideraram suficientes (normais) os teores foliares associados ao intervalo de índice DRIS de $-6,67 \leq \mathrm{I} \leq 6,67$, ao passo que, neste trabalho, a faixa de teor de nutriente estabelecida como alta está relacionada com a amplitude de $0,0 \leq \mathrm{I} \leq 6,67$.

Espera-se que faixas de suficiência de menor amplitude permitam maior sensibilidade no diagnóstico do estado nutricional da cultura, uma vez que evitam equívocos decorrentes de interpretação como suficiente de um nutriente que realmente esteja em deficiência ou em excesso. No caso específico da soja, ressalta-se também que as classes de suficiência estabelecidas em Embrapa Soja et al. (2010) são praticamente as mesmas, em relação àquelas definidas por Sfredo et al. (1986), a partir das médias dos teores, definidas para seis regiões norte-americanas produtoras de soja (Indiana, Michigan, Minnesota, Missouri, Ohio e Wisconsin), apresentadas por Peck (1979). Assim, pode-se inferir que há possibilidade de melhoria do diagnóstico do estado nutricional da soja, adequando-se as faixas de suficiência para as condições regionais de solo, clima e potencial genético apresentado pelas variedades atualmente cultivadas.

Destaca-se, ainda, a preocupação quanto ao fato de se adotar, comumente, as mesmas faixas de suficiência para amostras constituídas de terceiro trifólio a partir do ápice (Malavolta et al., 1997; Embrapa Soja et al., 2010), bem como deste, acompanhado de pecíolos (Bataglia et al., 1996; Ribeiro et al., 1999). No intuito de avaliar a magnitude da importância de se considerar o tipo de amostra foliar sobre a interpretação da diagnose do estado nutricional, efetuou-se a determinação de teores de nutrientes em 96 amostras foliares de soja, coletadas sem e com pecíolo. Constatou-se que os teores de $\mathrm{Ca}, \mathrm{Mg}, \mathrm{S}$ e $\mathrm{Cu}$ independem de a amostra ser constituída apenas pelo limbo foliar, ou incluir, também, o pecíolo (Tabela 5). Con- tudo, quando se coleta o limbo foliar, os teores médios de N, P, B, Fe, Mn e Zn são significativamente superiores e os teores de K são significativamente inferiores, em comparação com os valores observados para amostras com pecíolo. O maior teor de K no pecíolo já era esperado, por causa da sua elevada concentração no tecido que lhe serve de conexão ao caule, denominado pulvino (Hanway $\&$ Weber, 1971). Por outro lado, nutrientes que se acumulam no limbo foliar tendem a estar presentes em menores concentrações, caso a amostra inclua o pecíolo.

Em suma, verifica-se que as diferenças existentes entre os teores de nutrientes em função do tipo de amostra foliar coletada, podem induzir a equívocos na diagnose foliar. Quando os teores médios determinados em amostras foliares sem ou com pecíolo (Tabela 5) são interpretados conforme Embrapa Soja et al. (2010), cujas faixas de suficiência são apresentadas na Tabela 4, constata-se que o tipo de folha índice amostrado está associado à discordância na diagnose do estado nutricional de $\mathrm{K}, \mathrm{S}, \mathrm{Cu}, \mathrm{Mn}$ e $\mathrm{Zn}$. Assim, observa-se que, mesmo para nutrientes em que não há diferenças significativas no teor em limbo foliar, comparado com o teor em trifólio com pecíolo, como no caso do $\mathrm{S}$ e $\mathrm{Cu}$, ainda há a possibilidade de equívoco no diagnóstico nutricional. Por outro lado, se os teores médios das 96 amostras de limbo foliar são interpretados conforme Embrapa Soja et al. (2010), e os teores médios das amostras de folhas com pecíolo são comparados com os valores estimados pelo método Índice DRIS (Tabela 4), verifica-se a existência, ainda, de diagnoses discordantes para K, Ca e Zn. Este resultado pode estar associado ao fato de que as faixas de suficiência estabelecidas em Embrapa Soja et al. (2010) mantiveram-se praticamente inalteradas nas últimas duas décadas, reforçando a necessidade de atualização desses valores de referência, para as condições edafoclimáticas da região Central do Brasil e o potencial genético das variedades cultivadas atualmente.

Por esta razão, efetuou-se ajuste de modelos de regressão, para os teores de nutrientes no limbo foliar, em função dos teores no trifólio com pecíolo (Tabela 6). A partir das equações de regressão lineares apresentadas

Tabela 5. Teores médios de nutrientes em 96 amostras foliares ${ }^{1}$ de soja (com ou sem pecíolo), coletadas em cinco municípios de Mato Grosso do Sul, nos anos agrícolas 2001/2002 a 2004/2005

\begin{tabular}{lcccccc}
\hline \multirow{2}{*}{ Tipo de folha } & $\mathbf{N}$ & $\mathbf{P}$ & $\mathbf{K}$ & $\mathbf{C a}$ & $\mathbf{M g}$ & $\mathbf{S}$ \\
\cline { 2 - 6 } & \multicolumn{7}{c}{$\mathrm{g} \mathrm{kg}^{-1}$} \\
\hline Sem pecíolo & 54,0 & 3,5 & 20,4 & 12,0 & 3,7 & 4,4 \\
Com pecíolo & $39,7^{* *}$ & $3,0^{* *}$ & $25,8^{* *}$ & $12,2^{\text {ns }}$ & $3,6^{\text {ns }}$ & $4,0^{\text {ns }}$ \\
\hline & $\mathbf{B}$ & $\mathbf{C u}$ & $\mathbf{F e}$ & $\mathbf{M n}$ & $\mathbf{Z n}$ \\
\cline { 2 - 6 } & \multicolumn{7}{c}{116} \\
Sem pecíolo & 48 & 15 & 132 & 116 & 52 \\
Com pecíolo & $43^{* *}$ & $14^{\mathrm{ns}}$ & $102^{* *}$ & $90^{* *}$ & $39^{* *}$ \\
\hline
\end{tabular}

${ }^{1}$ Terceiro trifólio totalmente formado, a partir do ápice, no ramo vegetativo da planta, coletado no estádio de floração plena. Para cada nutriente, ${ }^{\text {ns }} \mathrm{e} * *$ indicam diferenças não significativas e significativas a $1 \%$, respectivamente, pelo teste t. 
na Tabela 6 e dos valores estabelecidos para amostras com pecíolo (Tabela 4), estimaram-se as faixas de teores considerados adequados para amostras foliares sem pecíolo (Tabela 7). A comparação dos valores de referência, obtidos para os dois tipos de amostras foliares, permite constatar maiores teores de N, P, B, Fe e Zn, nos limites inferior e superior da faixa suficiente, estimados para o limbo foliar (Tabela 7), em relação àqueles estimados para trifólios com pecíolo (Tabela 4). Neste contexto, destaca-se o fato de que, para N, o limite superior, estimado para a faixa considerada suficiente em folhas com pecíolo (46,9 $\mathrm{g} \mathrm{kg}^{-1}$ de N, Tabela 4), é menor do que o limite inferior, estimado para esta mesma classe de teor, em folhas sem pecíolo (50,6 $\mathrm{g} \mathrm{kg}^{-1}$ de $\mathrm{N}$, Tabela 7). Assim, constata-se que, em uma amostra de folha de soja com $49 \mathrm{~g} \mathrm{~kg}^{-1} \mathrm{de} \mathrm{N}$, haveria o diagnóstico de teor baixo ou alto, considerando-se os valores de referência estimados para limbo foliar ou para folhas com pecíolo, respectivamente.
Ao se interpretar os teores médios das 96 amostras de limbo foliar e de folhas com pecíolo (Tabela 5), conforme os valores estimados nas Tabelas 7 e 4, respectivamente, constata-se a plena concordância do diagnóstico do estado nutricional com a classificação de N, P, Mg, B, Fe e Zn como suficientes e, os demais nutrientes, em níveis altos. Salienta-se ainda que, pela comparação da classe de teor suficiente, estimada para os nutrientes em amostras de limbo foliar (Tabela 7), com aquela estabelecida em Embrapa Soja et al. (2010), pode-se verificar, exceto para $\mathrm{N}$ e Zn, diminuição na amplitude de valores.

Diante do exposto, sugere-se a adoção de novas faixas de suficiência, estimadas a partir do método DRIS, para os Estados de Mato Grosso do Sul e Mato Grosso, para amostras foliares de algodoeiro e de soja. Acreditase que essas faixas de suficiência permitirão maior exatidão da avaliação do estado nutricional, à medida que evitam falsos diagnósticos, decorrentes da menor amplitude

Tabela 6. Equações de regressão para teores de macro $\left(\mathrm{g} \mathrm{kg}^{-1}\right)$ e micronutrientes $\left(\mathrm{mg} \mathrm{kg}^{-1}\right)$ no terceiro trifólio ${ }^{1}$ (TT) em função do teor no terceiro trifólio com pecíolo (TTP), em plantas de soja

\begin{tabular}{|c|c|c|}
\hline \multicolumn{2}{|c|}{ Equaçãó $^{2}$} & \multirow{2}{*}{$\frac{\mathbf{R}^{\mathbf{2}}}{0,761}$} \\
\hline$\hat{\mathrm{N}}_{\mathrm{TT}}=7,738+1,165 * * \mathrm{~N}_{\mathrm{TTP}}$ & $28,1 \leq \mathrm{N}_{\mathrm{TTP}} \leq 48,7 \mathrm{~g} \mathrm{~kg}^{-1}$ & \\
\hline$\widehat{\mathrm{P}}_{\mathrm{TT}}=0,407+1,032 * * \mathrm{P}_{\mathrm{TTP}}$ & $1,9 \leq \mathrm{P}_{\mathrm{TTP}} \leq 4,8 \mathrm{~g} \mathrm{~kg}^{-1}$ & 0,950 \\
\hline$\hat{\mathrm{K}}_{\mathrm{TT}}=2,287+0,701 * * \mathrm{~K}_{\mathrm{TTP}}$ & $15,0 \leq \mathrm{K}_{\mathrm{TTP}} \leq 33,5 \mathrm{~g} \mathrm{~kg}^{-1}$ & 0,917 \\
\hline$\hat{\mathrm{C}} \mathrm{a}_{\mathrm{TT}}=-1,125+1,075^{* *} \mathrm{Ca} \mathrm{a}_{\text {TTP }}$ & $5,1 \leq \mathrm{Ca}_{\mathrm{TTP}} \leq 29,1 \mathrm{~g} \mathrm{~kg}^{-1}$ & 0,987 \\
\hline$\hat{\mathrm{Mg}} \mathrm{g}_{\mathrm{TT}}=0,031+1,033 * * \mathrm{Mg}_{\mathrm{TTP}}$ & $1,8 \leq \mathrm{Mg}_{\text {TTP }} \leq 6,4 \mathrm{~g} \mathrm{~kg}^{-1}$ & 0,986 \\
\hline$\widehat{\mathrm{S}}_{\mathrm{TT}}=0,202+1,032 * * \mathrm{~S}_{\mathrm{TTP}}$ & $0,2 \leq \mathrm{S}_{\mathrm{TTP}} \leq 8,8 \mathrm{~g} / \mathrm{kg}$ & 0,979 \\
\hline$\hat{\mathrm{B}}_{\mathrm{TT}}=1,734+1,079 * * \mathrm{~B}_{\mathrm{TTP}}$ & $21 \leq \mathrm{B}_{\mathrm{TTP}} \leq 78 \mathrm{mg} \mathrm{kg}^{-1}$ & 0,960 \\
\hline$\widehat{\mathrm{C}} \mathrm{u}_{\mathrm{TT}}=1,442+0,992 * * \mathrm{Cu}_{\mathrm{TTP}}$ & $4 \leq \mathrm{Cu}_{\mathrm{TTP}} \leq 27 \mathrm{mg} \mathrm{kg}^{-1}$ & 0,965 \\
\hline$\widehat{\mathrm{Fe}} \mathrm{e}_{\mathrm{TT}}=1,747+1,276^{* *} \mathrm{Fe}_{\mathrm{TTP}}$ & $57 \leq \mathrm{Fe}_{\mathrm{TTP}} \leq 292 \mathrm{mg} \mathrm{kg}^{-1}$ & 0,976 \\
\hline$\widehat{\mathrm{M}} \mathrm{n}_{\mathrm{TT}}=2,231+1,260 * * \mathrm{Mn}_{\mathrm{TTP}}$ & $22 \leq \mathrm{Mn}_{\mathrm{TTP}} \leq 349 \mathrm{mg} \mathrm{kg}^{-1}$ & 0,994 \\
\hline$\widehat{\mathrm{Z}} \mathrm{n}_{\mathrm{TT}}=-2,017+1,377 * * \mathrm{Zn}_{\mathrm{TTP}}$ & $11 \leq \mathrm{Zn}_{\mathrm{TTP}} \leq 77 \mathrm{mg} \mathrm{kg}^{-1}$ & 0,946 \\
\hline
\end{tabular}

${ }^{1}$ Terceiro trifólio e terceiro trifólio com pecíolo totalmente formado, a partir do ápice, no ramo vegetativo da planta, coletado no estádio de floração plena. ${ }^{2}$ Modelos ajustados a partir de 96 amostras foliares de soja coletadas em lavouras comerciais e parcelas experimentais, em cinco municípios de Mato Grosso do Sul, nos anos agrícolas de 2001/2002 a 2004/2005.

Tabela 7. Teores de nutrientes estimados a partir do método Índice DRIS, para amostras foliares de soja constituídas de terceiro trifólio ${ }^{1}$ sem pecíolo, para a interpretação dos resultados da análise foliar

\begin{tabular}{lcccc}
\hline \multirow{2}{*}{ Nutriente } & Baixo & Suficiente & Alto & Excesso \\
\cline { 2 - 5 } & \multicolumn{5}{c}{$\mathrm{g} \mathrm{kg}^{-1}$} \\
$\mathrm{n}$ & $<50,6$ & 50,6 a 56,5 & 56,6 a 62,3 & $>62,3$ \\
$\mathrm{P}$ & 2,8 a 3,3 & 3,4 a 3,9 & $>3,9$ \\
$\mathrm{~K}$ & $<2,8$ & 14,4 a 17,2 & $>20,3$ \\
$\mathrm{Ca}$ & 6,2 a 8,9 & 9,0 a 11,5 & $>11,5$ \\
$\mathrm{Mg}$ & $<6,4$ & 3,9 a 4,9 & $>4,9$ \\
$\mathrm{~S}$ & $<3,0$ & 3,0 a 3,8 & 3,0 a 3,3 & $>3,3$ \\
& $<2,4$ & 2,4 a 2,9 & & $>56$ \\
$\mathrm{~B}$ & & $\mathrm{mg} \mathrm{kg}^{-1}$ & & $>12$ \\
$\mathrm{Cu}$ & $<37$ & 37 a 46 & 47 a 56 & $>155$ \\
$\mathrm{Fe}$ & 7 a 9 & 10 a 12 & $>97$ \\
$\mathrm{Mn}$ & $<7$ & 112 a 155 & $>78$ \\
$\mathrm{Zn}$ & $<77$ & 64 a 97 & 57 a 78 & \\
\hline
\end{tabular}

${ }_{1}$ Terceiro trifólio totalmente formado, a partir do ápice, no ramo vegetativo da planta, coletado no estádio de floração plena. 
de valores da classe de teor suficiente e, no caso específico da soja, da possibilidade de se distinguirem as diferenças existentes entre tipos de amostras foliares.

\section{CONCLUSÕES}

O ajuste de modelos de regressão para teores de nutrientes, em folhas de algodoeiro e de soja, relacionados com os respectivos Índices DRIS, considerando-se uma amplitude de índice DRIS de - $6,7 \leq \mathrm{I} \leq 0,0$, permite a estimativa da faixa de teores suficientes, com amplitude em geral menor do que aquela estabelecida para essas culturas.

Os valores de referência para a diagnose do estado nutricional da soja são influenciados pelo tipo de folha amostrada. O terceiro trifólio apresenta teores maiores de N, P, B, Fe, Mn e Zn e menores de K, em relação aos do terceiro trifólio com pecíolo.

Para a cultura da soja, há necessidade de estabelecimento de faixas de suficiência específicas para o tipo de folha índice amostrado.

\section{REFERÊNCIAS}

Alvarez V VH \& Leite R de A (1999) Fundamentos estatísticos das fórmulas usadas para cálculo dos índices DRIS. Boletim Informativo da Sociedade Brasileira de Ciência do Solo, 24:20-25.

Bataglia OC, Dechen AR \& Santos WR dos (1996) Princípios da diagnose foliar. In: Alvarez V VH, Fontes LEF \& Fontes MPF (Eds.). O solo nos grandes domínios morfoclimáticos do Brasil e o desenvolvimento sustentado. Viçosa, SBCS: UFV, DPS. p.647-660.

Beaufils ER (1973) Diagnosis and recommendation integrated system (DRIS). Pietermaritizburg, University of Natal. 132p. (Soil science bulletin, 1).

Embrapa Soja (2002) Tecnologias de produção de soja - Paraná 2003. Londrina. 195p. (Sistemas de produção, 2).

Embrapa Soja, Embrapa Cerrados \& Embrapa Agropecuária Oeste (2008) Tecnologias de produção de soja - região Central do Brasil 2008. Londrina. 280p. (Sistemas de produção, 12).

Embrapa Soja, Embrapa Cerrados \& Embrapa Agropecuária Oeste (2010) Tecnologias de produção de soja - região Central do Brasil 2011. Londrina. 255p. (Sistemas de produção, 14).

Fehr WR \& Caviness CE (1977) Stages of soybean development. Ames, Iowa State University. 12p.

Hanway JJ \& Weber CR (1971) N, P, and K percentages in soybean (Glycine $\max (\mathrm{L}$.$) Merrill) plants parts. Agronomy Journal,$ 63:286-290.

Harger N (2008) Faixas de suficiência para teores foliares de nutrientes em soja, definidas pelo uso do método DRIS, para solos de origem basáltica. Tese de Doutorado. Universidade Estadual de Londrina, Londrina. $88 \mathrm{p}$.

Kurihara CH (2004) Demanda de nutrientes pela soja e diagnose de seu estado nutricional. Tese de Doutorado. Universidade Federal de Viçosa, Viçosa. 100p.

Malavolta E, Vitti GC \& Oliveira SA de (1997) Princípios, métodos e técnicas de avaliação do estado nutricional. In: Malavolta E, Vitti GC \& Oliveira SA de (Eds.) Avaliação do estado nutricional da plantas: princípios e aplicações. 2.ed. Piracicaba, POTAFOS. p.115-230.
Marur CJ \& Ruano O (2001) A reference system for determination of developmental stages of upland cotton. Revista brasileira de oleaginosas e fibrosas, 5:313-317.

Oliveira SA de \& Cassol JJ (1995) Níveis de suficiência no solo e nas folhas para a soja no Município de Campo Novo do Parecís - MT. In: $25^{\circ}$ Congresso Brasileiro de Ciência do Solo, Viçosa. Resumos expandidos, SBCS/UFV. p.562-563.

Peck TR (1979) Plant analysis for production agriculture. In: $7^{\text {th }}$ Soil Plant Analysis Workshop, Bridgetown. Proceedings, Bridgetown. p.1-45.

Reis Junior R dos A \& Monnerat PH (2003) DRIS norms validation for sugarcane crop. Pesquisa Agropecuária Brasileira, 38:379385 .

Reis Junior R dos A (2002) DRIS norms universality in the corn crop. Communications in Soil Science and Plant Analysis, 33:711-735.

Ribeiro AC, Guimarães PTG \& Alvarez VVH (Eds.) (1999) Recomendações para o uso de corretivos e fertilizantes em Minas Gerais: $5^{\text {a }}$ aproximação. Viçosa, Comissão de Fertilidade do Solo do Estado de Minas Gerais. 359p.

Serra AP, Marchetti ME, Vitorino ACT, Novelino JO \& Camacho MA (2010) Determinação de faixas normais de nutrientes no algodoeiro pelos métodos CHM, CND e DRIS. Revista Brasileira de Ciência do Solo, 34:105-113.

Sfredo GJ, Lantmann AF, Campo RJ \& Borkert CM (1986) Soja: nutrição mineral, adubação e calagem. Londrina, EmbrapaCNPSo. 51p. (Documentos, 17).

Staut, LA, Kurihara CH (2001) Calagem e adubação. Dourados, Embrapa Agropecuária Oeste. p.103-123.

Urano EOM, Kurihara CH, Maeda S, Vitorino ACT, Gonçalves MC \& Marchetti ME (2007) Determinação de teores ótimos de nutrientes em soja pelos métodos Chance Matemática, Sistema Integrado de Diagnose e Recomendação e Diagnose da Composição Nutricional. Revista Brasileira de Ciência do Solo, 31:63-72. 